\title{
Trastornos del equilibrio en los niños
}

Dr. Aliro Correa U.

\begin{abstract}
The purpose of this papaer is the analysis of $2 \%$ children with vestibular syroptoms.

In the first group (144 patientsi the principul cumplaint was vertigo. After the neurulugic and otoneurologic study, it appears that epilepsy and bening paroxismal vertigu was the main cause of vertigo in this group.

In the second group composed by 117 children, the priocipal complaint was unsteadiness caused by cerebellar ur brain stem patholngy.

The third group (35 children) accused as the most frequent symplon, dizziness. The absence of alteration in the otoneurolingical examination was the rule.
\end{abstract}

Con relativa frecuencia el médico pediatra se ve enfrentado al problema del niño con trastornos del equilibrio.

Como muchas veces le es dificultuso su manejo. este trabajo intenta una orientación al respecto.

En la literatura mundial se acepta que alrededor del $1 \%$ de las consultas de un Servicio de Neurología infantil se debe a una alteración del equilibrio os a un síndrome vertiginnss propiamente tal. 1 ,

En esta publicación se analizan 296 casos con este problema, los cuales fueron atendidos en un lapso de 18 años, haciendo la salvedad que el autor no trabaja en un hospital infantil.

La primera dificultad que presenta el médico es la calificación del sintoma inicial. ya que generalmente el niño no lo puede definir con exactitud.

Para realizar este análisis, se dividió a los pacientes en tres grupos, de acuerdo al motivo de consulta.

\section{Sintoma inicial}

Alteraciones

Edad N. ${ }^{\circ}$ de casos Vertigo de la marcha Mareo

\begin{tabular}{rrrrr}
\hline $3-5$ & 32 & 2 & 9 & 21 \\
$6-10$ & 90 & 29 & 49 & 12 \\
$11-15$ & 174 & 113 & 59 & 2 \\
\hline 296 & 144 & 117 & 35
\end{tabular}

Servicuo Otorrinalaringologia Hosp. Salvador, Fac. Med. U. de Chile. Sede Oriente.
Debido a que no se encontró diferencia entre el número de los niños y niñas, en lo sucesivo no se menciona el sexo del enfermo.

En la tabla 1 se encuentra resumida la distribución de los pacientes de acuerdo a la edad y al motivo de consulta. Como se puede apreciar, ambos parámetros son orientadores, ya que la distribución de acuerdo a los mismos es diferente. Tabla 1.- Distribución por edad y por sintoma inicial en 296 niños con trastornos del equilibio.

Estamos conscientes que el sintoma "mareo" es vago e impreciso, sio embargo, no fue posible aclararlo más.

Nunca hemns atendido un nin̄o menur de tres añoz por estos problemas. Los mayores de quince años nose consideran, ya que desde el punto de vista que se analiza, su manejo es similar al del adulto.

La correlación entre la sintomatología, el examen neurológico y el examen funcional del octavo par nos parece interesante, por lo que se analizarán a continuación.

Para ello se han separado los pacientes de acuerdo al síntoma inicial. En lo que respecta al examen neurológico, se consigna como alterado aquellus casos en que a pesar de presentar anormalidades, el neurílogo no precisio el diagnóstico.

El primer grupı está compuesto por los niños que tuvieron un síndrome vertiginoso. El resumen de los hallazgos aparece en la tabla 2. 
Tabla 2-- Correlation entre el síntoma vertigo. el examen neurolingica y olológicon en 144 niñess con traslornes del equilibrio.

N." de

pacientes E. Neurológico E. Otológico

\begin{tabular}{rll}
\hline 25 & Epilepsia & Nonmal \\
44 & Nomal & Vértigo posicional \\
40 & Normal & Nomal \\
13 & Normal & Parálisis vestibular \\
6 & Normal & Intoxicación por drogas \\
5 & Hipertensión & Sindrome de linea \\
& endocraneana & media de fosa \\
3 & Normal & posterior \\
7 & Normal & Heudrops laberintico \\
1 & Jaqueca & Normal \\
\hline
\end{tabular}

Parece ser que el sindrome vertiginoso es ocasionado en la mayoría de los casos por un daño a nivel periférico, por lo que el examen otológico es muy importante en el establecimiento del diagnóstico.

En nuestra casuística, fue útil en el 55\% de los casos.

La alteración neurológica más frecuente es la disritmia electroencelalográfica, que fue catalogada como epilepsia por los neurólogos en 25 niños.

Es importante hacer notar que sólo dos de éstos nin̄os registraba episodios convulsivos, sin embargo. los 23 restantes, luego de la crisis vertiginosa presentaban episodios de somnolencia de varias horas de duración.

Si a estos niños sumamos el paciente que fue calificado como jaquecoso, podemos establecer que el diagnóstico final fue realizado por el examen neurológico en el $18 \%$ de los casos.

En cinco niños (3\%) el diagnóstico se realizó en forma conjunta por el neurólogo y el otorrinólogo. determinándose la presencia de un síndrome de linea media de fosa posterior. Finalmente en 40 pacientes (27\%) no se pudo definir la causa de su sindrome vertiginoso, lo que debe estimular al planeamiento de estudios más acuciusos.

Esta misma correlación se realizó entre los niños que presentaban alteraciunes en la marcha. obteniéndose los resultados que se observan en la tabla 3.

Tabla 3.- Correlación entre el sintoma alteración de la marcha.

El examen neurolígico y otolígico en 117 niños con transtornos del equilibrio.

$$
\text { N." de }
$$

pacientes E. Neurológico E. Utológico

\begin{tabular}{|c|c|c|}
\hline 45 & Alterado & $\begin{array}{l}\text { Sindrome de linea mediat } \\
\text { de fosa posterior }\end{array}$ \\
\hline 16 & $\begin{array}{l}\text { Síndrome } \\
\text { cerebeloso }\end{array}$ & $\begin{array}{l}\text { Sindromit } \\
\text { cerebeloso }\end{array}$ \\
\hline 26 & Normal & Normal \\
\hline 16 & Alterado & Normal \\
\hline 7 & Alterado & $\begin{array}{l}\text { Sindrome de aingulo pun- } \\
\text { to cerebeloso. }\end{array}$ \\
\hline 4 & Alterado & Romboencefalitis \\
\hline 3 & Nomal & $\begin{array}{l}\text { Neuronitis } \\
\text { vestibular }\end{array}$ \\
\hline
\end{tabular}

En este grupo llama poderosamente la atención la alta incidencia de anormalidad neurolígica, (75\%), lo que indudablemente coloca a este examen en el primer lugar en cuanto a rendimiento. Sin embargo, en el $48 \%$ de estos casos, el examen otológico también aportó información útil.

En este grupo encontramos un $22 \%$ de exámenes normales y sólo un $2,5 \%$ de patología otológica pura. lo que evidentemente ensombrece el pronóstico.

El tercer conjunto de niños es el más reducido, ya que sólo encontramos 35 pacientes. En la tabla 4 se resumen los hallazgos neurológicos y utológicos.

N. ${ }^{\circ}$ de

pacientes E. Neurológico E. Otulógico

\begin{tabular}{rll}
\hline 15 & Normal & Normal \\
5 & Nornal & Vértigo posicional \\
4 & Normal & Neuronitis restibular \\
2 & $\begin{array}{l}\text { Epilepsia } \\
3\end{array}$ & $\begin{array}{l}\text { Sindrome } \\
\text { cerebeloso }\end{array}$ \\
4 & $\begin{array}{l}\text { Síndrome } \\
\text { cerebeloso }\end{array}$ & Sindrome de línea media \\
1 & $\begin{array}{l}\text { Hipertensión } \\
\text { endoraneana } \\
\text { Normal }\end{array}$ & $\begin{array}{l}\text { Síndrome de ángulo pun- } \\
\text { to cerebeloso } \\
\text { Parálisis vestibular }\end{array}$ \\
\hline
\end{tabular}

Llama la atención el alto número de exámenes normales, por lo que al parecer confirma lo expuesto por Eviatar,(2) quien señala que el mareo es un sintoma muy inespecifico $y$ por to tanto poco orientador.

El 28\% de los casos presentó un examen neurolígico positivo, mientras que el examen otológico ayudó prácticamente en el $50 \%$ de ellos.

\section{DISCUSION:}

Del análisis de nuestro material se desprenden 
Yarius hechos importanter. El primern es la necesidad de precisar ol lermino "mareo". ya que detrás de él se esconden tantos casos banales como ratos graves.

Por esu, creo cue nuestra clasificacion. basady en la anamnesis es útil. ya que permite diferenciar tres grupos de niños con diferente enfocpue clinicon. diagnóstice y pronéstico.

También podemos afirmar que. frente a un niño ron trastornus del equilibrio, debemos realizar siempre un examen neurológiro con E.E.G., y un examen olorrinolaringológico con examen funcional del octavo par. Con estus procedimientos obtenemos un diagnústico en el $72 \%$ de los casos.

Llama la atención cuán frecuente es la epilepsia en el grupo de niños con alteración neurológica (21\%).

Esto es especialmente válido en el grupo de pacientes con síndrome vertiginoso.

Es importante recalcar las características de la disritmia. la cual adquiere ciertos atributos clínicos peculiares.
El examen otorrinolaringylogico es fundamental cuando existe un informe neuruligiso normal. Este nos permite realizar una loscalización topográfi'a de la lesion y muchas veces tambićn nos precisa una etiologia.

Cuando existe una alteracioin en la marcha, el rol fundamental lo juega el neurílogo, ya rue el examen otoligico resulta ser soilı un auxiliar más en el logro del diagnistico final.

Por último es necesario destacar que existe un grupo importante de niñog (alrededor del $30 \%$ ) en el cual no se logra precisar la causa de las molestias.

Es mi muy personal impresión que en estos niños existe un trastorno conductual ode adaptación evidente, situación que debiera ser investigada por el especialista adecuado.

\section{REFERENCIAS}

I Beddoe, G.: "Vertigo in child hood". The otolaryngologic clinics of North America, 10: 139-144, $197 \overline{7}$.

"Eviatar, L., y Eviatar, A.: "Vertigo in child hood". Clinical pediatrics. 13: 940-942, 1974. 